\title{
Hydrodynamics in a Symmetric Random Medium
}

\author{
J. Fritz ${ }^{\star}$
}

Mathematical Institute, H.A.S., H-1364 Budapest, Pf. 127, Hungary, and Institut des Hautes Etudes Scientifiques, F-91440 Bures-sur-Yvette, France

\section{Dedicated to Roland Dobrushin}

\begin{abstract}
We investigate the hydrodynamic behaviour of a one-dimensional Ginzburg-Landau model with conservation law in the presence of random conductivities. It is found that there is no interference between nonlinearity and randomness; the conductivities average out in the same way as they do in the case of the underlying random walk in the given medium. This means that we have an effective conductivity specified as the harmonic mean of microscopic conductivities. Some extensions including multidimensional systems in a small electric field are also discussed.
\end{abstract}

\section{Introduction}

The idea that it might be possible to derive the macroscopic laws of hydrodynamics from microscopic principles by mathematical methods goes back to Morrey [11]. A general reformulation of the problem in terms of infinite systems and Gibbs states was presented by Dobrushin [3], see [1, 2, 4, 13] for the first results concerning some more or less explicitly solved models. General methods are available for stochastic models only, all tractable systems are at least subordinated to a reversible model, see $[6-9]$ and $[5,10]$. The main purpose of this paper is to investigate such models where the deterministic part of the microscopic current is not the gradient of a potential. In the case of random conductivities even a formal identification of the limiting equation is somewhat problematic. As in our previous papers, we apply some basic tools of the theory of parabolic equations. A different, very effective approach was proposed simultaneously by Varadhan [17].

Let us consider a one-dimensional lattice system of continuous spins $\omega_{k} \in \mathbb{R}$, $k \in \mathbb{Z}$. The evolution is defined by

$$
\begin{gathered}
d \omega_{k}=\frac{1}{2} c_{k}\left[V^{\prime}\left(\omega_{k+1}\right)-V^{\prime}\left(\omega_{k}\right)\right] d t+\frac{1}{2} c_{k-1}\left[V^{\prime}\left(\omega_{k-1}\right)-V^{\prime}\left(\omega_{k}\right)\right] d t \\
+\sqrt{c_{k-1}} d w_{k-1}-\sqrt{c_{k}} d w_{k}, \quad \omega_{k}(0)=\sigma_{k}, \quad k \in \mathbb{Z}
\end{gathered}
$$

* Supported in part by: Hungarian National Foundation for Scientific Research Grant No. 1815, National Science Foundation Grant DMR 8612369 
where $V: \mathbb{R} \rightarrow \mathbb{R}$ is a convex potential, $w_{k}, k \in \mathbb{Z}$ is a family of independent standard Wiener processes, and $c_{k}, k \in \mathbb{Z}$ is a fixed set of positive numbers. In the physical picture, see [6] for further references, $V^{\prime}$ is a chemical potential, thus $c_{k}$ should be interpreted as the conductivity between sites $k$ and $k+1$. In the present, onedimensional case $c_{k}$ may be very arbitrary, we only need a law of large numbers for $1 / c_{k}$, the resistance. We are interested in the limiting behaviour of the rescaled density field $S_{t}^{\varepsilon}\left(\varphi, \sigma_{\varepsilon}\right)$ as $\varepsilon \rightarrow 0$,

$$
S_{t}^{\varepsilon}\left(\varphi, \sigma_{\varepsilon}\right)=\int \varphi(x) \omega_{t}^{\varepsilon}(x) d x, \quad \omega_{t}^{\varepsilon}(x)=\omega_{[x / \varepsilon]}\left(t / \varepsilon^{2}\right), \quad \sigma_{\varepsilon}=\omega_{0}^{\varepsilon},
$$

where $[u]$ denotes the integer part of $u \in \mathbb{R}$. This problem can be exposed as follows. Let $c_{\varepsilon}(x)=c_{[x / \varepsilon]}, w_{t}^{\varepsilon}(x)=\varepsilon w_{[x / \varepsilon]}\left(t / \varepsilon^{2}\right)$, and introduce a difference operator, $\nabla_{\varepsilon}$, and its adjoint by

$$
\begin{gathered}
\nabla_{\varepsilon} \varphi(x)=\varepsilon^{-1}[\varphi(x+\varepsilon)-\varphi(x)], \quad \nabla_{\varepsilon}^{*} \varphi(x)=\varepsilon^{-1}[\varphi(x-\varepsilon)-\varphi(x)], \\
d \omega_{t}^{\varepsilon}=-\frac{1}{2} \nabla_{\varepsilon}^{*} c_{\varepsilon} \nabla_{\varepsilon} V^{\prime}\left(\omega_{t}^{\varepsilon}\right) d t+\nabla_{\varepsilon}^{*} \sqrt{c_{\varepsilon}} d w_{t}^{\varepsilon} \\
d S_{t}^{\varepsilon}=d M_{t}^{\varepsilon}-\frac{1}{2} \int\left(\nabla_{\varepsilon} \varphi(x)\right) c_{\varepsilon}(x) \nabla_{\varepsilon} V^{\prime}\left(\omega_{t}^{\varepsilon}(x)\right) d x d t \\
M_{t}^{\varepsilon}=\int\left(\nabla_{\varepsilon} \varphi(x)\right) \sqrt{c_{\varepsilon}(x)} w_{t}^{\varepsilon}(x) d x
\end{gathered}
$$

The martingale part $M^{\varepsilon}$ vanishes as $\varepsilon \rightarrow 0$, but there is an interplay between this vanishing randomness and the growing singularity of the drift part. As a consequence, $V^{\prime}\left(\omega^{\varepsilon}\right)$ converges only in a weak $\mathbb{L}^{2}$ sense; this is a characteristic feature of all hydrodynamic limit problems. On the other hand, $c_{\varepsilon}$ is assumed to be a rapidly oscillating function, thus we have to evaluate a product of weakly convergent functions, $c_{\varepsilon}$ and $\nabla_{\varepsilon} V^{\prime}\left(\omega^{\varepsilon}\right)$; both converge in an $\mathbb{H}^{-1}$ topology. In such situations it is not really surprising that the limit of the product shall not coincide with the product of limits. We shall show that the behaviour of this product is essentially the same as in the trivial case of $V^{\prime}(x)=x$, when the problem reduces to the central limit theorem for the related random walk in medium $c$. More exactly, if $p_{c}(t, j, k)$ denotes the transition probability of the random walk with generator $\mathbb{L}=-(1 / 2) \nabla_{1}^{*} c \nabla_{1}$, and $\varrho_{k}$ is the mean value of $\omega_{k}$ given the medium, then (1.1) implies $\varrho_{k}(t)=\Sigma \varrho_{j}(0) p_{c}(t, j, k)$. Therefore the diffusive scaling (1.2) results in a limiting equation $\partial \varrho / \partial t=(\bar{c} / 2) \partial^{2} \varrho / \partial x^{2}$, where $\bar{c}$ is the effective diffusion constant of the random walk. For example, if $c_{k}$ is an ergodic sequence, then $\bar{c}$ is just the harmonic mean of $c$, that is $1 / \bar{c}=\left\langle 1 / c_{k}\right\rangle$. This simply means that the resistance, the additive quantity averages out in a direct way. The general case happens to be quite similar, an equivalent hydrodynamic behaviour is obtained by replacing $c_{k}$ by the effective conductivity, $\bar{c}$ above. A more convincing explanation of this situation is presented at the end of the next section.

\section{Formulation of the Problem and Main Result}

The law of large numbers we are going to prove for the rescaled field $S^{\varepsilon}$ can be formulated in the most natural way in terms of a scale of weighted $\mathbb{L}^{2}$ spaces, $\mathbb{L}_{r}^{2}$, $r \in \mathbb{R}$. The basic configuration space is $\mathbb{L}_{e}^{2}$ defined as the locally convex space

$$
\mathbb{L}_{e}^{2}=\bigcap_{r>0} \mathbb{L}_{r}^{2}, \quad \mathbb{L}_{e}^{2 *}=\bigcup_{r<0} \mathbb{L}_{r}^{2}
$$


where $\mathbb{L}_{r}^{2}$ denotes the Hilbert space of locally integrable $u: \mathbb{R} \rightarrow \mathbb{R}$ with norm $|\cdot|_{r},|u|_{r}^{2}=\langle u, u\rangle_{r},\langle u, v\rangle_{r}=\int \theta_{r}(x) u(x) v(x) d x, \theta_{r}(x)=(\theta(x))^{r}$, and $\theta: \mathbb{R} \rightarrow(0,1]$ is determined by $\theta(x)=1$ if $|x| \leqq 1, \theta^{\prime}(x) / \theta(x)=-\operatorname{sign} x$ if $|x| \geqq 3$, and $\theta^{\prime}(x) / \theta(x)$ $=-(x-\operatorname{sign} x) / 2$ if $1 \leqq|x| \leqq 3 . \theta_{r}$ is a smooth version of $e^{-r|x|} . \mathbb{L}_{e}^{2}$ is a reflexive space, and $\mathbb{L}_{e}^{2 *}$ is its dual with respect to the usual scalar product $\langle\cdot, \cdot\rangle_{0}$. The spaces of absolutely continuous $u: \mathbb{R} \rightarrow \mathbb{R}$ such that $u, u^{\prime} \in \mathbb{L}_{e}^{2}$, or $u, u^{\prime} \in \mathbb{L}_{e}^{2 *}$, will be denoted by $\mathbb{H}_{e}^{1}$ and $\mathbb{H}_{e}^{1 *}$, respectively.

In view of (1.1)-(1.3) we consider $\omega_{t}^{\varepsilon}$ as a Markov process in $\Omega_{\varepsilon}$, the space of $u \in \mathbb{L}_{e}^{2}$ such that $u(x)=u(\varepsilon[x / \varepsilon])$; the distribution of $\omega_{t}^{\varepsilon}$ is a Borel probability concentrated od $\Omega_{\varepsilon}$. Suppose that $\mu_{\varepsilon}, \varepsilon>0$ is a family of probability measures on $\mathbb{L}_{e}^{2}$ such that $\mu_{\varepsilon}\left(\Omega_{\varepsilon}\right)=1$, and observe that if $\varphi \in \mathbb{L}_{e}^{2 *}$, then $\varphi(\sigma)=\int \varphi(x) \sigma(x) d x$ is $\mu_{\varepsilon}$-a.s. a normalized sum. We say that $\mu_{\varepsilon}$ satisfies the law of large numbers with asymptotic mean $\varrho \in \mathbb{L}_{e}^{2}$ if $\varphi(\sigma) \rightarrow \varphi(\varrho)$ in probability as $\varepsilon \rightarrow 0$, for all $\varphi \in \mathbb{L}_{e}^{2 *}$. If $\mu_{\varepsilon}$ is tight, then this is equivalent to $\int g(\sigma) d \mu_{\varepsilon} \rightarrow g(\varrho)$ whenever $g: \mathbb{L}_{e}^{2} \rightarrow \mathbb{R}$ is weakly continuous and bounded. This motivates the role played by the weak topology of $\mathbb{L}_{e}^{2}$ in the proofs.

Our conditions on the evolution are listed as follows. $V: \mathbb{R} \rightarrow \mathbb{R}$ has three continuous derivatives, $V^{\prime \prime \prime}$ is bounded, and we have a constant $\alpha \in(0,1)$ such that $\left|V^{\prime \prime}(x)-1\right| \leqq \alpha$ for all $x \in \mathbb{R}$. The medium is specified for each $\varepsilon>0$ by some $c_{\varepsilon} \in \Omega_{\varepsilon}$ such that $\left|c_{\varepsilon}(x)-1\right| \leqq \alpha$ for $x \in \mathbb{R}$, and there exists a continuously differentiable $\bar{c}: \mathbb{R} \rightarrow[1-\alpha, 1+\alpha]$ such that $\bar{c}^{\prime}$ is also bounded, and

$$
\lim _{\varepsilon \rightarrow 0} \int \phi(x) / c_{\varepsilon}(x) d x=\int \phi(x) / \bar{c}(x) d x \text { for } \phi \in \mathbb{H}_{e}^{1 *} .
$$

The limiting equation can be described as follows. As explained in $[6,7,10]$, due to some large deviation effects of the vanishing martingale part of $d S_{t}^{\varepsilon}$, the microscopic chemical potential $V^{\prime}$ should be replaced by its macroscopic average, $J^{\prime}=J^{\prime}(m)$, where $m=m(t, x)$ is the limiting density, and $J^{\prime}$ is the derivative of

$$
J(\varrho)=\sup _{\lambda}(\lambda \varrho-F(\lambda)), \quad F(\lambda)=\log \int e^{\lambda x-V(x)} d x
$$

Independent of this nonlinear effect, the conductivity averages out just as in the linear case, thus the equation for $m$ becomes

$$
\partial_{t} m=\frac{1}{2} \partial_{x}\left[\bar{c}(x) \partial_{x} J^{\prime}(m)\right]
$$

where $\partial_{t}$ and $\partial_{x}$ denote differentiation with respect to $t$ and $x$. Since $\bar{c}$ and $J^{\prime \prime}$ are both strictly positive, (2.4) is uniquely solved in the following sense, see e.g. [7]. For each $\varrho \in \mathbb{H}_{e}^{1}$ there exists a continuous trajectory, $m(t, \cdot)$ in $\mathbb{L}_{e}^{2}$ such that $m(0, \cdot)=\varrho, m(t, \cdot) \in \mathbb{H}_{e}^{1}$ a.s., $|m(t, \cdot)|_{r}^{2}$ is locally integrable for each $r>0$, and

$$
\partial_{t} \int \varphi(x) m(t, x) d x=-\frac{1}{2} \int \varphi^{\prime}(x) \bar{c}(x) \partial_{x} J^{\prime}(m(t, x)) d x \text { a.s. }
$$

whenever $\varphi \in \mathbb{H}_{e}^{1}$. Now we are in a position to state the main result; some extensions are to be discussed at the end of the paper.

Theorem. Suppose that the initial configuration, $\sigma_{\varepsilon}$ converges weakly in $\mathbb{L}_{e}^{2}$ to some $\varrho \in \mathbb{H}_{e}^{1}$ as $\varepsilon \rightarrow 0$. Then $S_{t}^{\varepsilon}\left(\phi, \sigma_{\varepsilon}\right) \rightarrow \int \phi(x) m(t, x) d x$ in probability for each $\phi \in \mathbb{H}_{e}^{1 *}$, where $m$ is the weak solution to $(2.5)$ with initial condition $m(0, \cdot)=\varrho$. 
Notice that the statement for $t>0$ is slightly weaker than the initial condition. The initial configuration need not be random, this is due to $V^{\prime \prime}>0$, cf. $[6,7,9,10]$. This suggests that there must be a local ergodic theorem behind. The proof follows the approach of $[6,7]$ with some modifications, there are three basic observations.

(i) The dynamics is so smooth that time averages like

$$
X_{z}^{\varepsilon}(\sigma, g)=\int_{0}^{\infty} e^{-z t} g\left(\omega_{t}^{\varepsilon}\right) d t, \quad z>0
$$

happen to be weakly continuous functions of the initial configuration $\sigma=\omega_{0}^{\varepsilon}$; this is due to the parabolic structure of (1.3). Let $\Sigma \subset \mathbb{L}_{e}^{2}$ be convex, and denote $\mathbb{C}_{w}(\Sigma)$ the space of weakly continuous and bounded maps of $\Sigma$ into $\mathbb{R}$. We say that $f \in \mathbb{C}_{w}(\Sigma)$ has a continuous functional derivative, $\mathbb{D} f$, if $\mathbb{D} f$ is a weakly continuous and bounded map of $\Sigma$ into $\mathbb{L}_{e}^{2 *}$ such that

$$
f(\sigma)-f(\bar{\sigma})=\int_{0}^{1} \int \delta(x) \operatorname{DD} f(x, \bar{\sigma}+s \delta) d x d s
$$

whenever $\sigma, \bar{\sigma} \in \Sigma, \delta=\sigma-\bar{\sigma}$. It is easy to check that $\mathbb{D} f$ is uniquely defined, the space of differentiable $f$ is denoted by $\mathbb{D}(\Sigma)$. Suppose now that $c \in \Omega_{\varepsilon},|c(x)-1| \leqq \alpha$, $a(t, \cdot) \in \Omega_{\varepsilon},|a(t, x)-1| \leqq \alpha$ for all $t$ and $x$, and denote $p_{a, c}^{\varepsilon}(s, x ; t, y)$ the fundamental solution to

$$
\partial_{t} u=-\frac{1}{2} \nabla_{\varepsilon}^{*} c \nabla_{\varepsilon} a u
$$

i.e. $p_{a, c}^{\varepsilon}(s, x ; s, y)=1 / \varepsilon$ if $[x / \varepsilon]=[y / \varepsilon]$, and it is 0 otherwise. Since $\varepsilon p_{a, c}^{\varepsilon}$ $=\partial \omega_{t}^{\varepsilon}(y) / \partial \omega_{s}^{\varepsilon}(x)$ if $c=c_{\varepsilon}$ and $a(t, y)=V^{\prime \prime}\left(\omega_{t}^{\varepsilon}(y)\right)$, we have $\mathbb{D} X_{z}^{\varepsilon}=\mathbb{P}_{a, c}^{z, \varepsilon} h_{0}$ with $\Sigma=\Omega_{\varepsilon}$ and $h_{t}(y)=\mathbb{D} g\left(y, \omega_{t}^{\varepsilon}\right)$, where

$$
\mathbb{P}_{a, c}^{z, \varepsilon} h_{s}(x)=\int_{s}^{\infty} e^{z s-z t} \int p_{a, c}^{\varepsilon}(s, x ; t, y) h_{t}(y) d y d t
$$

The range and the domain of this operator will be specified in the next section. It is absolutely crucial that $\mathbb{P}_{a, c}^{z, \varepsilon}$ admits some bounds that do not depend on the smoothness of the coefficients; in fact, they depend only on $\alpha$. Parabolic estimates of this kind allow us to conclude that $X^{\varepsilon}$ is weakly continuous, and this property is uniform in $\varepsilon>0$. Therefore, the law of large numbers applies also to $X^{\varepsilon}$, but this is not enough to reach a conclusion.

(ii) To identify the limit poits of $X^{\varepsilon}$, we have to control $g\left(\omega_{t}^{\varepsilon}\right)$ also for fixed times, which is the same as understanding the behaviour of the time averages of $\mathbb{G}_{\varepsilon} g\left(\omega_{t}^{\varepsilon}\right)$, where $\mathbb{G}_{\varepsilon}$ denotes the generator associated to our Markov process, $\omega_{t}^{\varepsilon}$. More exactly, if $f_{z}^{\varepsilon}(\sigma)=\mathbb{E}\left[X_{z}^{\varepsilon}(\sigma, g)\right]$, then we have a resolvent equation

$$
g(\sigma)=z f_{z}^{\varepsilon}(\sigma)-\mathbb{G}_{\varepsilon} f_{z}^{\varepsilon}(\sigma), \quad \sigma \in \Omega_{\varepsilon}, \quad z>0,
$$

at least if $g$ is a smooth cylinder function. Notice first that the resolvent equation for the semigroup generated by $(2.4)$ is just

$$
\begin{gathered}
g(\varrho)=z f_{z}(\varrho)+\frac{1}{2} \int\left[\partial_{x} J^{\prime}(\varrho(x))\right] \bar{c}(x) \partial_{x} \mathbb{D} f_{z}(x, \varrho) d x, \quad \varrho \in \mathbb{H}_{e}^{1} ; \\
\mathbb{G}_{\varepsilon} f(\sigma)=\frac{\varepsilon}{2} \int e^{V(\sigma(x))} \mathbb{D}\left[e^{-V(\sigma(x))} \nabla_{\varepsilon}^{*} c_{\varepsilon}(x) \nabla_{\varepsilon} \mathbb{D} f(x, \sigma)\right](x, \sigma) d x
\end{gathered}
$$


for smooth $f$ and $\sigma \in \Omega_{\varepsilon}$. We wanted to pass from (2.10) to (2.11). The factor $\varepsilon$ in (2.12) is certainly encouraging; the structural difference between (2.10) and (2.11) immediately disappears if we introduce random initial conditions, the family of local equilibrium distributions. For each $\varepsilon>0$ and $\lambda \in \Omega_{\varepsilon}$ we define a Borel probability, $\mu_{\lambda, \varepsilon}$ on $\mathbb{L}_{e}^{2}$ by postulating $\exp [\lambda(x) u-V(u)-F(\lambda(x))]$ as the Lebesgue density, $q_{x}(u)$ of the distribution of $\sigma(x) ; \sigma(x)=\sigma(y)$ if $[x / \varepsilon]=[y / \varepsilon]$; they are completely independent otherwise. Obviously, $\mu_{\lambda, \varepsilon}\left(\Omega_{\varepsilon}\right)=1$. This family plays a distinguished role because of the following symmetry property, see [6,7]. Let $\mu_{v, \varepsilon}^{0}$ denote the measure with $\lambda(x)=v$ for all $x \in \mathbb{R}$. Integrating by parts we see that $\mathbb{G}_{\varepsilon}$ is symmetric in $\mathbb{I}^{2}\left(\mu_{v, \varepsilon}^{0}\right)$, and

$$
\int f_{1} \mathbb{G}_{\varepsilon} f_{2} d \mu_{v, \varepsilon}^{0}=\frac{1}{2} \iint\left[\nabla_{\varepsilon} \mathbb{D} f_{1}(x, \sigma)\right] c_{\varepsilon}(x) \nabla_{\varepsilon} \mathbb{D} f_{2}(x, \sigma) d x d \mu_{v, \varepsilon}^{0},
$$

thus (2.10) can be rewritten into a weak form such that only one functional derivative is involved. Taking the expectation of both sides of (2.10), by (2.13) we get for all $\lambda \in \Omega_{\varepsilon}$ and smooth $g$

$$
\begin{aligned}
\int g(\sigma) \mu_{\lambda, \varepsilon}(d \sigma)= & z \int f_{z}^{\varepsilon}(\sigma) \mu_{\lambda, \varepsilon}(d \sigma) \\
& +\frac{1}{2} \iint\left(\nabla_{\varepsilon} \lambda(x)\right) c_{\varepsilon}(x) \nabla_{\varepsilon} \mathbb{D} f_{z}^{\varepsilon}(x, \sigma) d x \mu_{\lambda, \varepsilon}(d \sigma) .
\end{aligned}
$$

Since $\lambda$ is just as arbitrary as $\sigma,(2.10)$ and (2.14) are equivalent. The advantage of (2.14) consists in the fact that it is much less singular than (1.4), and the structure of (2.14) is essentially the same as that of the limiting equation (2.11). Indeed, we have an identity, $\varrho_{\varepsilon}(x)=\int \sigma(x) \mu_{\lambda, \varepsilon}(d \sigma)=F^{\prime}(\lambda(x))$, while the inverse function of $F^{\prime}$ is just $J^{\prime}$, i.e. $\lambda(x)=J^{\prime}\left(\varrho_{\varepsilon}(x)\right)$ revealing the structural similarity of (2.14) and (2.11). The weak equicontinuity of $\mathbb{D} f_{z}^{\varepsilon}$ and that of $\nabla_{\varepsilon} \mathbb{D} f_{z}^{\varepsilon}$ will be proven by means of parabolic inequalities, thus we can pass to (2.11) as soon as we understand how $c_{\varepsilon}$ averages out.

(iii) In view of (ii), for each $\varrho \in \mathbb{H}_{e}^{1}$ we must find a $\lambda \in \Omega_{\varepsilon}$ in such a way that $\mu_{\lambda, \varepsilon}$ satisfies the law of large numbers with asymptotic mean $\varrho$ as $\varepsilon \rightarrow 0$. Perhaps the most natural choice is $\lambda(x)=J^{\prime}\left(I_{\varepsilon} \varrho(x)\right)$, where $I_{\varepsilon} \varrho(x)$ denotes the integral mean of $\varrho$ over the interval $[\varepsilon[x / \varepsilon], \varepsilon+\varepsilon[x / \varepsilon])$. By means of a more sophisticated construction we can compensate the irregularity of the medium, too. In the one-dimensional case it is quite easy to choose $\lambda$ in such a way that $c_{\varepsilon} \nabla_{\varepsilon} \lambda$ be smooth. More exactly, define $\lambda \in \Omega_{\varepsilon}$ by

$$
c_{\varepsilon}(x) \nabla_{\varepsilon} \lambda(x)=\left(I_{\varepsilon} \bar{c}(x)\right) \nabla_{\varepsilon} J^{\prime}\left(I_{\varepsilon} \varrho(x)\right), \quad \lambda(0)=J^{\prime}\left(I_{\varepsilon} \varrho(0)\right),
$$

and denote $\mu_{\lambda, \varepsilon}, \varepsilon>0$ the associated family of product measures. It is easy to check that $\mu_{\lambda, \varepsilon}$ satifies the law of large numbers with asymptotic mean $\varrho$; this is the point where (2.2) will be exploited.

Now we are in a position to outline the main steps of the proof. Since the diffusion coefficient of (1.3) is a constant, while its drift is uniformly Lipschitz continuous, the existence and uniqueness of strong solutions in $\Omega_{\varepsilon}$ follows by the most standard iteration procedure, see [7] for further references. The same method implies also (2.10), at least for functions $g$ of type

$$
g(\sigma)=h\left(\varphi_{1}(\sigma), \varphi_{2}(\sigma), \ldots, \varphi_{n}(\sigma)\right), \quad \sigma \in \Omega_{\varepsilon},
$$


where $\varphi_{k} \in \mathbb{L}_{e}^{2 *}$ and $h: \mathbb{R}^{n} \rightarrow \mathbb{R}$ has two continuous derivatives with compact support. By means of the parabolic estimates of the next section we show that $f_{z}^{\varepsilon}$, $\mathbb{D} f_{z}^{\varepsilon}$ and $\nabla_{\varepsilon} \mathbb{D} f_{z}^{\varepsilon}$ are all uniformly bounded and weakly equicontinuous on the bounded sets of $\mathbb{L}_{e}^{2}$. Since the bounded sets are weakly precompact in $\mathbb{L}_{e}^{2}$, the Arzela-Ascoli theorem allows us to select uniformly convergent subsequences. In view of (ii) and (iii) we can now pass from (2.14) to (2.11) simultaneously for all $\varrho \in \mathbb{H}_{e}^{1}$. Since (2.11) is uniquely solved, we have

$$
\lim _{\varepsilon \rightarrow 0} \iint_{0}^{\infty} e^{-z t} \mathbb{P}_{\varepsilon}^{t} g(\sigma) d t \mu_{\lambda, \varepsilon}(d \sigma)=\int_{0}^{\infty} e^{-z t} g(m(t, \cdot)) d t
$$

where $\mathbb{P}_{\varepsilon}^{t} g(\sigma)=\mathbb{E}\left[g\left(\omega_{t}^{\varepsilon} \mid \omega_{0}^{\varepsilon}=\sigma\right]\right.$, and $m$ satisfies (2.4) with initial condition $\varrho$. Again by a parabolic inequality, we show that $\int \mathbb{P}_{\varepsilon}^{t} g(\sigma) d \mu_{\lambda, \varepsilon}$ is an equicontinuous function of $t$, at least for $g$ of type (2.16) with $\varphi_{k} \in \mathbb{H}_{e}^{1 *}$, which implies $\int \mathbb{P}_{\varepsilon}^{t} g d \mu_{\lambda, \varepsilon} \rightarrow g(m(t, \cdot))$ for such $g$. Now the final conclusion follows by showing that $\mathbb{P}_{\varepsilon}^{t} g(\sigma)$ is a weakly equicontinuous function of $\sigma$. Unfortunatelly, we are not able to show that the conditional distribution of $\omega_{t}^{\varepsilon}$ given $\omega_{t}^{\varepsilon}=\sigma$ remains tight as $\varepsilon \rightarrow 0$, thus we can not extend the statement for all weakly continuous $g$.

\section{Parabolic Estimates}

We consider the operators (2.9) in some weighted $\mathbb{L}^{q}$-spaces, $\mathbb{L}_{r}^{q}\left(\mathbb{R}_{+}^{2}\right)$. Let $\mathbb{R}_{+}^{2}=[0, \infty) \times \mathbb{R}, r \in \mathbb{R}, 1 \leqq q<\infty$, then $\mathbb{L}_{r}^{q}\left(\mathbb{R}_{+}^{2}\right)$ is defined as the space of locally integrable $h: \mathbb{R}_{+}^{2} \rightarrow \mathbb{R}$ with norm $\|\cdot\|_{r, q}$,

$$
\|h\|_{r, q}=\left|\int_{0}^{\infty}\left(\left|h_{t}\right|_{r, q}\right)^{q} d x\right|^{1 / q}, \quad|\sigma|_{r, q}=\left.\left.\left|\int \theta_{r}(x)\right| \sigma(x)\right|^{q} d x\right|^{1 / q}, \quad \sigma: \mathbb{R} \rightarrow \mathbb{R} .
$$

If $q=2$ then the second subscript will be omitted: $\|h\|_{r}=\|h\|_{r, 2}$.

Lemma 1. Suppose that $c \in \Omega_{\varepsilon}, a(t, \cdot) \in \Omega_{\varepsilon},|c(x)-1| \leqq \alpha$ and $|a(t, x)-1| \leqq \alpha$ for all $t \geqq 0$ and $x \in \mathbb{R}$. If $\alpha<1,|r \varepsilon| \leqq 1$ and $z \geqq C_{\alpha} r^{2}$ then for all $h \in \mathbb{L}_{r}^{2}\left(\mathbb{R}_{+}^{2}\right)$ we have

$$
z\left|\mathbb{P}_{a, c}^{z, \varepsilon} h_{0}\right|_{r}^{2}+(1-\alpha)\left\|\nabla_{\varepsilon} \mathbb{P}_{a, c}^{z, \varepsilon} h_{0}\right\|_{r}^{2} \leqq C_{\alpha}\|h\|_{r}^{2}
$$

where $C_{\alpha}$ is a universal constant depending only on $\alpha$.

Proof. Observe that $u_{s}=\mathbb{P}_{a, c}^{z, \varepsilon} h_{s}$ satisfies a backward equation,

$$
-\partial_{s} u+z u+\frac{a}{2} \nabla_{\varepsilon}^{*} c \nabla_{\varepsilon} u=h
$$

thus

whence

$$
-z \partial_{s}|u|_{r}^{2}+2 z^{2}|u|_{r}^{2}+z\left\langle u, a \nabla_{\varepsilon}^{*} c \nabla_{\varepsilon} u\right\rangle_{r}=2 z\langle u, h\rangle_{r}
$$

$$
z\left|u_{0}\right|_{r}^{2} \leqq\|h\|_{r}^{2}+\left\|\nabla_{\varepsilon}^{*} c \nabla_{\varepsilon} u\right\|_{r}^{2}
$$

On the other hand, $\nabla_{\varepsilon} u$ satisfies a self-adjoint equation. Since $\left|\nabla_{\varepsilon} \theta_{r}(x)\right| \leqq 3|r| \theta_{r}(y)$ if $|x-y| \leqq \varepsilon$ and $|r \varepsilon| \leqq 1$, we have an "integration by parts" formula $\left\langle\nabla_{\varepsilon} u, v\right\rangle_{r}$ 
$-\left.\left\langle u, \nabla_{\varepsilon}^{*} v\right\rangle_{r}|\leqq 3| r|| u\right|_{r}|v|_{r} ;$ thus by an easy calculation

whence

$$
\begin{aligned}
-\partial_{s}\left\langle\nabla_{\varepsilon} u, c \nabla_{\varepsilon} u\right\rangle_{r} & +2 z(1-\alpha)\left|\nabla_{\varepsilon} u\right|_{r}^{2}+(1-\alpha)\left|\nabla_{\varepsilon}^{*} c \nabla_{\varepsilon} u\right|_{r}^{2} \\
& \leqq 12|r|\left|\nabla_{\varepsilon} u\right|_{r}\left|\nabla_{\varepsilon}^{*} c \nabla_{\varepsilon} u\right|_{r}+12|r|\left|\nabla_{\varepsilon} u\right|_{r}|h|_{r} \\
& +2\left|\nabla_{\varepsilon}^{*} c \nabla_{\varepsilon} u\right|_{r}|h|_{r}
\end{aligned}
$$

$$
\left|\nabla_{\varepsilon} u_{0}\right|_{r}^{2}+\frac{1-\alpha}{2}\left\|\nabla_{\varepsilon}^{*} c \nabla_{\varepsilon} u\right\|_{r}^{2} \leqq C_{\alpha}^{\prime}\|h\|_{r}^{2}
$$

which completes the proof by an easy calculation.

This lemma bounds $\mathbb{D} X_{z}^{\varepsilon}$ and $\nabla_{\varepsilon} \mathbb{D} X_{z}^{\varepsilon}$, thus the weak equicontinuity of $X_{z}^{\varepsilon}$ will follow by the next statement, see Lemma 6 of [7].

Lemma 2. For each bounded $B \subset \mathbb{L}_{e}^{2}, \beta>0, r>0$ and $K<+\infty$ there exists a weak neighborhood $U$ of 0 in $\mathbb{L}_{e}^{2}$ such that $|\varphi(\delta)|<\beta$ whenever $\varphi \in \Omega_{\varepsilon},|\varphi|_{-r}+\left|\nabla_{\varepsilon} \varphi\right|_{-r} \leqq K$ and $\delta \in U \cap B$.

The weak equicontinuity of $\nabla_{\varepsilon} \mathbb{D} f$ is more problematic. Let $\bar{a}(t, y)=V^{\prime \prime}\left(\bar{\omega}_{t}^{\varepsilon}(y)\right)$, where $\bar{\omega}^{\varepsilon}$ denotes the solution with initial configuration $\bar{\sigma} \in \Omega_{\varepsilon}, \delta=\sigma-\bar{\sigma}$. From (3.2)

$$
\mathbb{P}_{a, c}^{z, \varepsilon} h=\mathbb{P}_{\bar{a}, c}^{z, \varepsilon} h+\mathbb{P}_{\bar{a}, c}^{z, \varepsilon}\left(\frac{a-\bar{a}}{2} \nabla_{\varepsilon}^{*} c \nabla_{\varepsilon} \mathbb{P}_{a, c}^{z, \varepsilon} h\right)
$$

while $a-\bar{a}$ vanishes in a mean sense as $\delta \rightarrow 0$, thus we need

Lemma 3. Under conditions of Lemma 1 we have some $q_{\alpha}>2$ and $C_{\alpha}<+\infty$ such that if $z \geqq C_{\alpha} r^{2},|r \varepsilon| \leqq 1$ and $2 \leqq q<q_{\alpha}$, then

$$
\left\|\nabla_{\varepsilon}^{*} c \nabla_{\varepsilon} \mathbb{P}_{a, c}^{z, \varepsilon} h\right\|_{r, q} \leqq C_{\alpha}\|h\|_{r, q} \text { for all } h \in \mathbb{L}_{r}^{q}\left(\mathbb{R}_{+}^{2}\right)
$$

Proof. Let $\mathbb{Q}_{c}^{\varepsilon}=\nabla_{\varepsilon}^{*} c \nabla_{\varepsilon} \mathbb{P}_{1, c}^{z, \varepsilon}$ and denote $q_{c}^{\varepsilon}$ its kernel. If $c=1$ then we have an explicit expression:

$$
\begin{gathered}
q_{1}^{\varepsilon}(s, x ; t, y)=\left(\frac{1}{\varepsilon}\right)^{3} e^{z s-z t} I\left(t \varepsilon^{-2}-s \varepsilon^{-2},[y / \varepsilon]-[x / \varepsilon]\right) \\
I(t, n)=\frac{1}{\pi} \int_{-\pi}^{+\pi}(1-\cos \varphi) e^{-t}(1-\cos \varphi) \cos n \varphi d \varphi
\end{gathered}
$$

Integrating by parts and using $\varphi^{2} / 5 \leqq 1-\cos \varphi \leqq \varphi^{2} / 2$, we obtain that $|I(t, n)|$ $\leqq C\left(t n^{4}\right)^{-1 / 2}$ if $n \neq 0,\left|\partial_{t} I(t, 0)\right| \leqq C t^{-2}$. On the other hand,

$$
\begin{aligned}
\mathbb{Q}_{1}^{\varepsilon} h_{s}(x)= & \int_{s}^{\infty} \int_{[y / \varepsilon]=[x / \varepsilon]} q_{1}^{\varepsilon}(s, x ; t, y) h_{t}(y) d y d t \\
& +\varepsilon^{-2} \int_{s}^{\infty} I\left(t \varepsilon^{-2}-s \varepsilon^{-2}, 0\right) I_{\varepsilon} h_{t}(x) d t .
\end{aligned}
$$

The first term can be estimated directly by the Young inequality, while a basic theorem on Hilbert transforms applies in the second case, see Theorem 1 of 
Chap. 2.2 in [15]. We obtain that $\left\|\mathbb{Q}_{1}^{\varepsilon}\right\|_{0, q}<+\infty$ for each $q>1$, and the bound does not depend on $\varepsilon$. Since $\left\|\mathbb{Q}_{1}^{\varepsilon}\right\|_{0}=2$ by the Plancherel theorem, the Riesz-Thorin interpolation theorem yields some $q_{\alpha}>2$ such that $\alpha\left\|\mathbb{Q}_{1}^{\varepsilon}\right\|_{0, q}<2$ if $2 \leqq q<q_{\alpha}$. Therefore, choosing $a=c, \bar{a}=c=1$ in the perturbation formula (3.5), we get $\left\|\Delta_{\varepsilon} \mathbb{P}_{c, 1}^{z, \varepsilon}\right\|_{0, q}<+\infty$ if $q \in\left[2, q_{\alpha}\right)$, where $\Delta_{\varepsilon}=-\nabla_{\varepsilon}^{*} \nabla_{\varepsilon}=-\nabla_{\varepsilon} \nabla_{\varepsilon}^{*}$. Introduce now $K_{\varepsilon}$, the inverse of $\nabla_{\varepsilon}$ in $\Omega_{\varepsilon} \cap \mathbb{L}_{e}^{2 *}$,

$$
K_{\varepsilon} \sigma(x)=\varepsilon \sum_{k=-\infty}^{n-1} I_{\varepsilon} \sigma(\varepsilon k), \quad n=[x / \varepsilon] .
$$

The adjoint of $K_{\varepsilon}$ is defined by

$$
K_{\varepsilon}^{*} \sigma(x)=\varepsilon \sum_{k=n}^{\infty} I_{\varepsilon} \sigma(\varepsilon k), \quad n=[x / \varepsilon]+1 .
$$

Therefore, if $\Omega_{\varepsilon}^{*}=\Omega_{\varepsilon} \cap \mathbb{L}_{e}^{2 *}$, then the last bound of $\Delta_{\varepsilon} \mathbb{P}_{c, 1}^{z, \varepsilon}$ yields

$$
\begin{gathered}
\left\|\nabla_{\varepsilon} \mathbb{Q}_{c}^{\varepsilon} K_{\varepsilon} h\right\|_{0, q} \leqq 2 C_{\alpha}^{\prime}\|h\|_{0, q}, \\
\left\|K_{\varepsilon}^{*} \mathbb{Q}_{c}^{\varepsilon} \nabla_{\varepsilon}^{*} h\right\|_{0, q} \leqq 2 C_{\alpha}^{\prime}\|h\|_{0, q} \quad \text { if } \quad h \in \Omega_{\varepsilon}^{*}, \quad 2 \leqq q<q_{\alpha} .
\end{gathered}
$$

In view of (3.8)-(3.9), we wanted to bound $Q_{c}^{\varepsilon}$ by means of the Stein interpolation theorem, see Theorem 4.1 of Chap. $V$ in [16].

$$
\mathbb{R}_{v}=\left(-\Delta_{\varepsilon}\right)^{-v} \nabla_{\varepsilon} Q_{c}^{\varepsilon} K_{\varepsilon}\left(-\Delta_{\varepsilon}\right)^{v}, \quad 0 \leqq \operatorname{Re} v \leqq 1
$$

is an analytic family of operators, thus the conditions of Stein's theorem reduce to

$$
\begin{aligned}
& \left|\left(-\Delta_{\varepsilon}\right)^{i y} \sigma\right|_{0, q} \leqq M(y)|\sigma|_{0, q}, \\
& \sup _{y \in \mathbb{R}} e^{-|y|} \log M(y)<\infty \text { for } \sigma \in \Omega_{\varepsilon}, q \in\left[2, q_{\alpha}\right) .
\end{aligned}
$$

This problem is essentially independent of $\varepsilon,\left(-\Delta_{1}\right)^{i y}$ can be represented as convolution with $q=q(n), n \in \mathbb{Z}$, where

$$
q(n)=(2 \pi)^{-1} \int_{-\pi}^{+\pi}(1-\cos \phi)^{i y} e^{i n \phi} d \phi
$$

that is, we are faced with a discrete version of the Hilbert transform. Integrating by parts we see that

$$
\left(1+n^{2}\right)|q(n+1)-q(n)| \leqq\left(1+y^{2}\right)\left\{C+\left|\int_{-\pi}^{+\pi} g(\phi) h(\phi) \sin n \phi d \phi\right|\right\}
$$

where $h(\phi)=\sin \phi(1-\cos \phi)^{i y-1}$, while $g$ is smooth. Observe that $|h(\phi)| \leqq 5 /|\phi|$, $\left|h^{\prime}(\phi)\right| \leqq 10(1+|y|) \phi^{-2}$, and the integrand is bounded by $C^{\prime}|n|$ on $(-\pi / n, \pi / n)$, thus integrating by parts on the remainder we obtain that $\left(1+n^{2}\right)|q(n+1)-q(n)|$ $\leqq C^{\prime \prime}(1+|y|)^{3}$, whence we obtain a universal constant $\bar{C}$ such that

$$
\sum_{n \in A^{m}}|q(n-m)-q(n)| \leqq \bar{C}(1+|y|)^{3} \quad \text { for all } m \in Z,
$$

where $A^{m}$ is the set of $\mathrm{n} \in Z$ such that $|n| \geqq 2|m|$. 
Since $\left|(1-\cos \phi)^{i y}\right| \leqq 2$, we have just verified the conditions of the Corollary to Theorem 1 of Chap. 2.2 in [15], which implies $\left|\left(-\Delta_{\varepsilon}\right)^{i y}\right|_{0, q} \leqq C(1+|y|)^{3}$ for all $1<q<\infty$; thus we have (3.11). Now we are in a position to apply Stein's theorem, we obtain an $\mathbb{L}^{q}$-bound for $\mathbb{R}_{1 / 2}$. On the other hand, the kernel of $\left(-\Delta_{\varepsilon}\right)^{-1 / 2} \nabla_{\varepsilon}$ and that of its inverse, $K_{\varepsilon}\left(-\Delta_{\varepsilon}\right)^{1 / 2}$, are explicitly calculable, and each of them happens to be invariant under scaling. For $\varepsilon=1$ we have convolutions with $q(n)=C(1+2 n)^{-1}$ and $q(n)=C(1-2 n)^{-1}$, thus the above remark on the discrete Hilbert transform implies the statement for $a=1$ and $r=0$.

Thus the proof can be completed by interpolation and perturbation. We apply first the Riesz-Thorin theorem to get $\alpha\left\|\mathbb{Q}_{c}^{\varepsilon}\right\|_{0, q}<2$ for small $q>2$. Then the perturbation identity (3.5) yields the statement for general coefficients, $a$, but we still have $r=0$. Since (3.4) solves this problem for each $r$ with $q=2$, the final statement follows now by a direct application of Stein's theorem.

Consider now the initial distributions $\mu_{\lambda, \varepsilon}$ with $\lambda=\lambda_{\varepsilon}$ defined by (2.15). They are product measures with uniformly bounded variances, thus the law of large numbers reduces to the asymptotic behaviour of the mean values, $F^{\prime}\left(\lambda_{\varepsilon}\right)$.

Lemma 4. If $\varrho \in \mathbb{H}_{e}^{1}$ then $\lim F^{\prime}\left(\lambda_{\varepsilon}\right)=\varrho$ in $\mathbb{L}_{e}^{2}$ as $\varepsilon$ goes to 0 .

Proof. Observe first that both $J^{\prime}$ and $F^{\prime}$ possess strictly positive and bounded first derivatives. Let $\bar{K}_{\varepsilon} \sigma(x)=K_{\varepsilon} \sigma(x)-K_{\varepsilon} \sigma(0)$; it extends to a bounded map of $\mathbb{L}_{e}^{2}$ into itself, see (3.6). We have $\lambda_{\varepsilon}(x)=J^{\prime}\left(I_{\varepsilon} \varrho(x)\right)+\delta_{\varepsilon}(x), \delta_{\varepsilon}(x)=\bar{K}_{\varepsilon} \sigma(x)$ with $\sigma=\left(\bar{c}(x) / c_{\varepsilon}(x)\right) \nabla_{\varepsilon} J^{\prime}\left(I_{\varepsilon} \varrho(x)\right)$. Equation (2.2) implies immediately

$$
\lim _{\varepsilon \rightarrow 0} \int \phi(x)\left\{1-\bar{c}(x) / c_{\varepsilon}(x)\right\} d x=0 \quad \text { for all } \phi \in \mathbb{L}^{1}(\mathbb{R})
$$

and (3.15) remains in force even if $\phi$ varies in a compact subset of $\mathbb{L}^{1}(\mathbb{R})$; therefore both $\delta_{\varepsilon}$ and $\nabla_{\varepsilon} \delta_{\varepsilon}$ go to zero weakly in $\mathbb{L}_{e}^{2}$ as $\varepsilon \rightarrow 0$, which completes the proof by the dominated convergence theorem.

As we shall show in the next section, the above results allow us to pass to the limiting resolvent Eq. (2.11); the time average can be removed by means of the following estimate.

Lemma 5. Let $a$ and $c$ be as in Lemma $1, u_{s}(x)=\int p_{a, c}^{\varepsilon}(s, x ; t, y) h_{t}(y) d y$ for $s \leqq t$, $18 r^{2} \leqq 1,|r \varepsilon| \leqq 1$, then

$$
\left|u_{0}\right|_{r}^{2}+(1-\alpha)\left|\nabla_{\varepsilon} u_{0}\right|_{r}^{2} \leqq\left\{\left|h_{t}\right|_{r}^{2}+2\left|\nabla_{\varepsilon} h_{t}\right|_{r}^{2}\right\} e^{t} .
$$

Proof. Since $\partial_{s} u+(a / 2) \nabla_{\varepsilon}^{*} c \nabla_{\varepsilon} u=0$, like in the proof of Lemma 1, we obtain

$$
\begin{aligned}
-\partial_{s}\left\{|u|_{r}^{2}\right. & +\left\langle\nabla_{\varepsilon} u, c \nabla_{\varepsilon} u\right\rangle_{r} \leqq|u|_{r}\left|a \nabla_{\varepsilon}^{*} c \nabla_{\varepsilon} u\right|_{r}+|6 r|\left|\sqrt{c} \nabla_{\varepsilon} u\right|_{r}\left|\sqrt{a} \nabla_{\varepsilon}^{*} c \nabla_{\varepsilon} u\right|_{r} \\
& -\left|\sqrt{a} \nabla_{\varepsilon}^{*} c \nabla_{\varepsilon} u\right|_{r}^{2} \leqq|u|_{r}^{2}+\left\langle\nabla_{\varepsilon} u, c \nabla_{\varepsilon} u\right\rangle_{r},
\end{aligned}
$$

which completes the proof.

The tools summarized above enable us to follow the lines of the proof of Theorem 1 of [7]. For the reader's convenience we reproduce the main steps in the next section in the present, notationally much simpler situation. 


\section{Proof of the Main Result}

We start with the derivation of (2.17); it is sufficient to consider functions $g$ of type $g_{\phi}(\sigma)=g(\phi(\sigma))$, where $\phi \in \mathbb{H}_{e}^{1}, \phi(\sigma)=\int \phi(x) \sigma(x) d x$, and $g: \mathbb{R} \rightarrow \mathbb{R}$ is bounded together with its first and second derivatives. We want to select uniformly convergent subsequences from each of $X_{z}^{\varepsilon}, \mathbb{D} X_{z}^{\varepsilon}, \nabla_{\varepsilon} \mathbb{D} X_{z}^{\varepsilon}$ as $\varepsilon \rightarrow 0$. Since the bounded sets are weakly precompact both in $\mathbb{L}_{e}^{2}$ and in $\mathbb{L}_{e}^{2 *}$, we have to show that $X_{z}^{\varepsilon}, \mathbb{D} X_{z}^{\varepsilon}$ and $\nabla_{\varepsilon} \mathbb{D} X_{z}^{\varepsilon}$ are all bounded and equicontinuous when $\sigma$ varies in a bounded set of $\mathbb{L}_{e}^{2} . X_{z}^{\varepsilon}$ is obviously bounded, while Lemma 1 applies to $\mathbb{D} X_{z}^{\varepsilon}$ and $\nabla_{\varepsilon} \mathbb{D} X_{z}^{\varepsilon}$. Their equicontinuity with respect to $z$ can be shown by estimating their derivatives. The case of $X_{z}^{\varepsilon}$ is again trivial, but $(t-s) p_{a, c}^{\varepsilon}(s, x ; t, y) e^{z s-z t}$ is just the kernel of the square of $\mathbb{P}_{a, c}^{z, \varepsilon}$; thus (3.14) and Lemma 1 yield bounds also for the derivatives of $\mathbb{D} X_{z}^{\varepsilon}$ and $\nabla_{\varepsilon} \mathbb{D} X_{z}^{\varepsilon}$ with respect to $z$.

Now we turn to the problem of continuous dependence on $\sigma \in B$, where $B$ is an arbitrary ball in $\mathbb{L}_{e}^{2} ; \sigma \in B$ means that $|\sigma|_{r} \leqq R(r)$ for all $r>0$ with some increasing, but finite $R:(0, \infty) \rightarrow(0, \infty)$. Let $\omega_{t}^{\varepsilon}$ and $\bar{\omega}_{t}^{\varepsilon}$ denote strong solutions to (1.3) with initial configurations $\sigma$ and $\bar{\sigma}, \quad \delta=\sigma-\bar{\sigma}, \quad \tilde{a}(t, y)=\left\{V^{\prime}\left(\omega_{t}^{\varepsilon}(y)\right)-V^{\prime}\left(\bar{\omega}_{t}^{\varepsilon}(y)\right)\right\}$ $\left.\left\{\omega_{t}^{\varepsilon}(y)-\bar{\omega}_{t}^{\varepsilon}(y)\right)\right\}^{-1}$; taking the difference of two copies of (1.3) with identical Wiener trajectories, for any $k \in \mathbb{L}_{r}^{2}\left(\mathbb{R}_{+}^{2}\right)$ with $r<0$ we get

$$
\int_{0}^{\infty} \int\left\{\omega_{t}^{\varepsilon}(y)-\bar{\omega}_{t}^{\varepsilon}(y)\right\} k_{t}(y) d y d t=\int \delta(x) \int_{0}^{\infty} \int p_{\tilde{a}, c}^{\varepsilon}(0, x ; t, y) k_{t}(y) d y d t d x .
$$

Let $k_{t}=e^{-z t}\left\{\omega_{t}^{\varepsilon}-\bar{\omega}_{t}^{\varepsilon}\right\}$, then by Lemma 1 for $z \geqq 2 C_{\alpha} r^{2}, r>0$,

$$
z \int_{0}^{\infty} e^{-z t}\left|\omega_{t}^{\varepsilon}-\bar{\omega}_{t}^{\varepsilon}\right|_{r}^{2} d t \leqq 2 C_{\alpha}|\delta|_{r}^{2}
$$

thus by Lemma 1 and Lemma 2 for each $\beta>0$ and $z \geqq 2 C_{\alpha} r^{2}$ we have a weak neighborhood $U=U_{\beta}^{z}(B)$ of $0 \in \mathbb{L}_{e}^{2}$ such that

$$
\int_{0}^{\infty} e^{-z t}\left|\omega_{t}^{\varepsilon}-\bar{\omega}_{t}^{\varepsilon}\right|_{r}^{2} d t \leqq \beta \quad \text { if } \quad \delta \in U \cap B,
$$

which implies immediately that $X_{z}^{\varepsilon}$ is weakly equicontinuous on each ball of $\mathbb{L}_{e}^{2}$.

The continuity problem of $\mathbb{D} X_{z}^{\varepsilon}$ and $\nabla_{\varepsilon} \mathbb{D} X_{z}^{\varepsilon}$ is more involved. From (3.5)

$$
\mathbb{D} X_{2 z}^{\varepsilon}\left(\sigma, g_{\phi}\right)-\mathbb{D} X_{2 z}^{\varepsilon}\left(\bar{\sigma}, g_{\phi}\right)=\mathbb{P}_{a, c}^{z, \varepsilon}(h-\bar{h})_{0}+\mathbb{P}_{\bar{a}, c}^{z,} \bar{k}_{0},
$$

where $a$ and $\bar{a}$ are as in (3.5), $h_{t}(y)=e^{-z t} \phi(y) g^{\prime}\left(\phi\left(\omega_{t}^{\varepsilon}\right)\right), \bar{h}$ is the same with $\bar{\omega}_{t}^{\varepsilon}$ in the place of $\omega_{t}^{\varepsilon}, 2 \bar{k}_{t}(y)=e^{-z t}\{a(t, y)-\bar{a}(t, y)\} \nabla_{\varepsilon}^{*} c \nabla_{\varepsilon} \mathbb{P}_{a, c}^{z, \varepsilon} \bar{h}_{t}(y)$. The weak equicontinuity of the first term and that of its gradient follows directly from (4.3) by Lemma 1, and the Laplace transform of $|a(t, \cdot)-\bar{a}(t, \cdot)|_{r}^{2}$ can be estimated in the same way. Suppose now that $|\phi|_{r, q}<\infty$ for some $r<0$ and $q>2$. Since $V^{\prime \prime}$ is bounded, Lemma 3 and the Hölder inequality imply that $\|\bar{k}\|_{r} \rightarrow 0$ for some $r<0$ as $\delta \in B$ vanishes in the weak sense, and this convergence is uniform in $\varepsilon>0$. Therefore, Lemma 1 implies that both $\mathbb{D} X_{z}^{\varepsilon}$ and $\nabla_{\varepsilon} \mathbb{D} X_{z}^{\varepsilon}$ are weakly equicontinuous on $B$, at least if $|\phi|_{r, q}<\infty$. In view of (4.2), this restriction can be removed quite easily by noticing that $\mathbb{D} X_{z}^{\varepsilon}\left(\sigma, g_{\phi}\right)-\mathbb{D} X_{z}\left(\bar{\sigma}, g_{\phi}\right)$ and $\nabla_{\varepsilon} \mathbb{D} X_{z}\left(\sigma, g_{\phi}\right)-\nabla_{\varepsilon} \mathbb{D} X_{z}\left(\bar{\sigma}, g_{\phi}\right)$ are uniformly continuous functions of $\phi \in \mathbb{L}_{e}^{2 *}$ for $\delta \in B$, which completes the proof of the equicontinuity problem, see [7] for technical details, if necessary. 
Now we are in a position to apply the Arzela-Ascoli theorem. Let $E=\cup B_{n}$ be a countable union of increasing balls, then we have a subsequence $\varepsilon_{n} \rightarrow 0$ such that each of $X_{z}^{\varepsilon}, \mathbb{D} X_{z}^{\varepsilon}, \nabla_{\varepsilon} \mathbb{D} X_{z}^{\varepsilon}$ converges on $E$ along this subsequence to some limit $X_{z}, Y_{z}, Z_{z}$. Moreover, the convergence is uniform on each $B_{n}$, the restrictions of the limiting objects to balls are still weakly continuous; thus it is easy to check, see (2.7), that $Y_{z}=\mathbb{D} X_{z}$ and $Z_{z}=\partial_{x} Y_{z}$. Consider now a countable union, $E^{\prime}=\cup B_{n}^{\prime}$ of balls of $\mathbb{H}_{e}^{1}$; we may assume that $\mu_{\lambda, \varepsilon}(E)=1$ for $\varrho \in E^{\prime}$. Using Lemma 4 and the associated law of large numbers, we see that each term of (2.14) converges to the corresponding term of (2.11) as $\varepsilon_{n} \rightarrow 0$, and each convergence relation holds true simultaneously for all $\varrho \in E^{\prime}$. Following the proof of Proposition 2 of [7] we see that the balls $B_{n}^{\prime}$ can be chosen to be so large that all solutions to (2.4) remain in $E^{\prime}$, consequently (2.11) has only one weakly continuous solution on $E$, which implies (2.17) for $\varrho \in E^{\prime}$, thus for all $\varrho \in \mathbb{H}_{e}^{1}$, too.

Consider now $m_{t}^{\varepsilon}\left(g_{\phi}\right)=\int \mathbb{P}_{\varepsilon}^{t} g_{\phi} d u_{\lambda, \varepsilon}$, and observe that

$$
\begin{gathered}
\partial_{t} m_{t}^{\varepsilon}\left(g_{\phi}\right)=\frac{1}{2} \iint\left(\nabla_{\varepsilon} \lambda_{\varepsilon}(x)\right) c_{\varepsilon}(x) \nabla_{\varepsilon} \mathbb{D}_{\varepsilon}^{t} g_{\phi}(x, \sigma) d x d \mu_{\lambda, \varepsilon}, \\
\mathbb{D} g_{\phi}\left(\omega_{t}^{\varepsilon}\right)(x, \sigma)=\int p_{a, c}^{\varepsilon}(0, x ; t, y) \phi(y) g^{\prime}\left(\phi\left(\omega_{t}^{\varepsilon}\right)\right) d y,
\end{gathered}
$$

thus $m_{t}^{\varepsilon}\left(g_{\phi}\right)$ is a bounded and equicontinuous function of time by Lemma 5 , consequently (2.17) implies $\lim m_{t}^{\varepsilon}\left(g_{\phi}\right)=g_{\phi}(m(t, \cdot))$ as $\varepsilon \rightarrow 0$, for each $t>0$, by the uniqueness of the Laplace transform. Finally, comparing (4.6), Lemma 5, Lemma 2 and (2.7), we obtain that $\lim \left\{g_{\phi}\left(\omega_{t}^{\varepsilon}\right)-g_{\phi}\left(\bar{\omega}_{t}^{\varepsilon}\right)\right\}=0$ whenever $\delta \rightarrow 0$ weakly in $\mathbb{L}_{e}^{2}$, which completes the proof as $\lim \mu_{\lambda, \varepsilon}[|\phi(\sigma)-\phi(\varrho)|>\beta]=0$ for each $\beta>0$ if $\varepsilon \rightarrow 0$.

\section{Concluding Remarks}

Our basic conditions $\left|V^{\prime \prime}(x)-1\right| \leqq \alpha$ and $\left|c_{\varepsilon}(x)-1\right| \leqq \alpha, \alpha<1$ can be replaced by $\alpha_{1}<V^{\prime \prime}(x)<\alpha_{2}, \alpha_{1}<c_{\varepsilon}(x)<\alpha_{2}, \alpha_{1}>0, \alpha_{2}<\infty$ simply by rescaling time with a constant factor. Of course, the rescaling changes the temperature of the system, but in the absence of phase transitions, this makes no difference.

The case of dimensions $d>1$ is a little bit more interesting. The form of (1.3) does not change; we define $\nabla_{\varepsilon}$ for $\phi: \mathbb{R}^{d} \rightarrow \mathbb{R}$ as a vector with components $\varepsilon^{-1}\left(\phi\left(x+\varepsilon e_{j}\right)\right.$ $-\phi(x))$, where $e_{1}, e_{2}, \ldots, e_{d}$ are the unit vectors of our orthogonal system of coordinates in $\mathbb{R}^{d}, \nabla_{\varepsilon}^{*}$ is defined for $\psi: \mathbb{R}^{d} \rightarrow \mathbb{R}^{d}$ by $\left\langle\nabla_{\varepsilon}^{*} \psi, \phi\right\rangle_{0}=\int\left(\psi, \nabla_{\varepsilon} \phi\right) d x$, $(\cdot, \cdot)$ and $\langle\cdot, \cdot\rangle_{0}$ denote the usual scalar products in $\mathbb{R}^{d}$ and $\mathbb{L}^{2}\left(\mathbb{R}^{d}\right)$, respectively. The extensions of Lemma 1, Lemma 2 and Lemma 5 are immediate, see [7]. Lemma 3 is less trivial because $\nabla_{\varepsilon}$ and $\nabla_{\varepsilon}^{*}$ do not commute if $d>1$, but I do not see any essential difficulty. The family of initial distributions, $\mu_{\lambda, \varepsilon}$ can be defined as follows. Assume that the associated random walk in the given medium $c_{\varepsilon}$ converges in the following sense: $\left|\mathbb{P}_{1, c}^{z, \varepsilon} h_{0}-\mathbb{P}_{1,1}^{z, \varepsilon} h_{0}\right|_{0} \rightarrow 0$ as $\varepsilon \rightarrow 0$ for $z>0$ and $h_{t}(y)=\phi(y)$ for all $t \geqq 0$ if $\phi$ is smooth. In view of the last step of the proof, see Sect. 4 , we may assume that both $\varrho$ and $\Delta \varrho$ belong to $\mathbb{L}^{2}\left(\mathbb{R}^{d}\right)$. In the spirit of [12], then it is quite natural to define $\lambda_{\varepsilon}$ as $\lambda_{\varepsilon}=\mathbb{P}_{1, c}^{\gamma, \varepsilon} h_{0}, h_{t}(y)=\Delta_{\varepsilon} J^{\prime}(\varrho(y)), \Delta_{\varepsilon}=-\nabla_{\varepsilon}^{*} \nabla_{\varepsilon}$, where $\gamma=\gamma(\varepsilon, \varrho) \rightarrow 0$ in a suitable way as $\varepsilon \rightarrow 0$. Notice that $\lambda_{\varepsilon}$ is just the solution of a resolvent equation,

$$
\gamma(\varepsilon, \varrho) \lambda_{\varepsilon}+\frac{1}{2} \nabla_{\varepsilon}^{*} c_{\varepsilon} \nabla_{\varepsilon} \lambda_{\varepsilon}=\frac{1}{2} \Delta_{\varepsilon} I_{\varepsilon} J^{\prime}(\varrho)
$$


where $I_{\varepsilon}$ is the integral mean over the Dirichlet cells of $\varepsilon \mathbb{Z}^{d}$, see [7]. From (5.1) we see that $\gamma\left|\lambda_{\varepsilon}\right|_{0} \rightarrow 0$ as $\gamma \rightarrow 0$, thus we can replace $c=c_{\varepsilon}$ in (2.14) by 1 . On the other hand, $\mathbb{P}_{1, c}^{\gamma, \varepsilon}-P_{1,1}^{\gamma, \varepsilon} \rightarrow 0$ for each fixed $\gamma>0$ as $\varepsilon \rightarrow 0$ by assumption, thus we can choose $\gamma=\gamma(\varepsilon, \varrho)$ in such a way that $\left|\lambda_{\varepsilon}-\bar{\lambda}_{\varepsilon}\right|_{0} \rightarrow 0$ as $\varepsilon \rightarrow 0$, where $\bar{\lambda}_{\varepsilon}$ is defined as the solution to $(5.1)$ with $c_{\varepsilon}=1$. Now an easy Fourier analysis shows that $\bar{\lambda}_{\varepsilon} \rightarrow J^{\prime}(\varrho)$ in $\mathbb{L}^{2}\left(\mathbb{R}^{d}\right)$ as $\varepsilon \rightarrow 0$, thus we can apply the law of large numbers to evaluate (2.14); we obtain $\partial_{t} m=(1 / 2) \Delta J^{\prime}(m)$ for the limiting density field. The case of macroscopically inhomogeneous conductivities is quite similar.

We have assumed only for simplicity that there is no interaction term in the energy $H(\sigma)=\Sigma V\left(\sigma_{k}\right)$. It is possible to replace $H$ by

$$
H_{b}(\sigma)=\sum_{k \in \mathbb{Z}^{d}}\left\{V\left(\sigma_{k}\right)+\sum_{|j-k|=1} b_{k j} U\left(\sigma_{k}-\sigma_{j}\right)\right\}
$$

where $V$ is as above, $b_{k j}=b_{j k}, U(x)=U(-x), b_{k j}$ and the matrix of second derivatives of $U$ are both strictly positive and bounded. The rescaled evolution law is obtained from (1.3) by replacing $V^{\prime}$ by $\partial H_{b}(x, \omega)=\partial H_{b} / \partial \omega(x)$, see [6,7] for the case of $b_{k j}=b, U=x^{2}$. Additional difficulties are technical; $F$, the effective free energy should be determined as a thermodynamical limit. If $b=b_{k j}$ is ergodic with respect to translations of $\mathbb{Z}^{d}$, then the subadditive ergodic theorem shows that $F$ is a deterministic function of the chemical potential $\lambda$; it depends only on the distribution of $b$; we obtain the same behaviour as before.

In the presence of a small electric field (1.3) turns into

$$
d \omega_{t}^{\varepsilon}=\nabla_{\varepsilon}^{*}\left\{e_{\varepsilon} c_{\varepsilon} B\left(\omega_{t}^{\varepsilon}\right)\right\} d t-\frac{1}{2} \nabla_{\varepsilon}^{*} c_{\varepsilon} \nabla_{\varepsilon} \partial H_{b}\left(\cdot, \omega_{t}^{\varepsilon}\right) d t+\nabla_{\varepsilon}^{*} \sqrt{c_{\varepsilon}} d w_{t}^{\varepsilon},
$$

where $e_{\varepsilon}: \mathbb{R}^{d} \rightarrow \mathbb{R}$ is uniformly bounded, and $B: \mathbb{R} \rightarrow \mathbb{B}$ has a bounded first derivative. The a priori bounds for the resolvent equation can be derived in much the same way as in the deterministic case of [8], thus we can follow the ideas outlined above. Instead of (5.1), now we define $\mu_{\lambda, \varepsilon}$ by

$$
\gamma(\varepsilon, \varrho) \lambda_{\varepsilon}-\nabla_{\varepsilon}^{*}\left\{e_{\varepsilon} c_{\varepsilon} \bar{B}\left(\lambda_{\varepsilon}\right)\right\}+\frac{1}{2} \nabla_{\varepsilon}^{*} c_{\varepsilon} \nabla_{\varepsilon} \lambda_{\varepsilon}=\frac{1}{2} \Delta_{\varepsilon} I_{\varepsilon} J^{\prime}(\varrho)-\nabla_{\varepsilon}^{*}\left\{\bar{e} I_{\varepsilon} \bar{B}\left(J^{\prime}(\varrho)\right),\right.
$$

where $\bar{B}\left(\lambda_{\varepsilon}\right)(x)=\int B(\sigma(x)) \mu_{\lambda, \varepsilon}(d \sigma)$, and $\bar{e}$ denotes the mean value of $e_{\varepsilon}, \bar{e}$ is assumed to be a constant. Since $\lambda_{\varepsilon} \rightarrow J^{\prime}(\varrho)$ seems to be true also in this case, we expect that $e_{\varepsilon}$, the electric field averages out in the usual way; thus there is no interaction between the randomness of the conductivities and that of the electric field; the effective conductivity remains unchanged.

Acknowledgements. An essential part of this work was done during my stay at the Center for Mathematical Sciences Research of Rutgers University. I am indebted to Joel L. Lebowitz for stimulating discussions and kind hospitality there. Remarks by Hermann Rost and Herbert Spohn have been also fruitful in the development of ideas. The paper has been finished at the institut des Hautes Etudes Scientifiques; I wish to express my thanks to Professor M. Berger for the kind hospitality.

\section{References}

1. Boldrighini, C., Dobrushin, R.L., Suhov, Yu.M.: The hydrodynamic limit of a degenerate model of statistical physics. Usp. Mat. Nauk 35, 152 (1980) (short communication, in Russian) 
2. Boldrighini, C., Dobrushin, R.L., Suhov, Yu.M.: One-dimensional hard rod caricature of hydrodynamics. Stat. Phys. 31, 577-616 (1983)

3. Dobrushin, R.L.: On the derivation of the equations of hydrodynamics. Lecture, Mathematical Institute, Budapest (1978)

4. Dobrushin, R.L., Siegmund-Schultze, R.: The hydrodynamic limit for systems of particles with independent evolution. Math. Nachr. 105, 199-244 (1982)

5. Donsker, M.D., Varadhan, S.R.S.: Large deviations from a hydrodynamic scaling limit. Preprint 1988.

6. Fritz, J.: On the hydrodynamic limit of a one-dimensional Ginzburg-Landau lattice model. The a priori bounds. Stat. Phys. 47, 551-572 (1987)

7. Fritz, J.: On the hydrodynamic limit of a Ginzburg-Landau lattice model. The law of large numbers in arbitrary dimensions. Preprint (1987). Probab. Theor. Rel. Fields 81, 291-318 (1989)

8. Fritz, J., Maes, Ch.: Hydrodynamic behaviour in a small external field. Preprint. Stat. Phys. 53, 1179-1205 (1988)

9. Funaki, T.: Derivation of the hydrodynamical equation for a 1-dimensional GinzburgLandau model. Preprint, to appear in Probab. Theor. Rel. Fields (1987)

10. Guo, M.Z. Papanicolau, G.C., Varadhan, S.R.S. (1988): Nonlinear diffusion limit for a system with nearest neighbor interactions. Commun. Math. Phys. 118, 31-59 (1988)

11. Morrey, C.: On the derivation of the equations of hydrodynamics from statistical mechanics. Commun. Pure. Appl. Math. 8, 279-327 (1955)

12. Papanicolau, G.C., Varadhan, S.R.S.: Boundary value problems with rapidly oscillating coefficients. In: Random Fields. Fritz, J., Lebowitz, J.L., Szász, D. (eds.) pp. 835-853. János Bólyai Mathematical Society and North Holland, Vol. II (1981)

13. Rost, H.: Non-equilibrium behaviour of a many particle system. Density profile and local equilibrium. Z. Wahrsch. Verw. Geb. 58, 41-55 (1981)

14. Reed, M., Simon, B.: Methods of modern mathematical physics II. Fourier analysis, SelfAdjointness. New York: Academic Press 1975

15. Stein, E.M.: Singular integrals and differentiability properties of functions. Princeton, NJ : Princeton University Press 1970

16. Stein, E.M., Weiss, G.: Introduction to Fourier analysis on Euclidean spaces. Princeton, NJ : Princeton University Press 1971

17. Varadhan, S.R.S.: In preparation

Communicated by Ya.G. Sinai

Received November 22, 1988 
